\title{
RETROSPECTIVE STUDY TO ASSESS AXILLARY MANAGEMENT AND ONCOLOGICAL OUTCOME AFTER NEOADJUVANT CHEMOTHERAPY FOR BREAST CANCER PATIENTS
}

Galal Abounaggah, Tarek El-Faiomy, Gehan khedr*, Mohamed Farouk Asal, Moataz Mohamed Ewedah Surgical Oncology Unit, Department of Surgery, Department of Clinical Oncology, Faculty of Medicine, Alexandria University, Alexandria, Egypt

\section{INTRODUCTION}

Preoperative chemotherapy (NAC) has been recognized as the standard of care for patients with locally advanced breast cancer and recently some patients with stage 2 and chemoresponsive subtypes (HER2, TNBC). Lymph node status is the most important prognostic factor in patients who receive neoadjuvant therapy. Patients who have a positive lymph node by fine-needle aspiration (or core needle biopsy) before he a postive ly . Primary tumor resection. ALND has been the standard treatment of the axilla after NAC for many years. SLNB as an alternative can reduce the extent of axillary surgery without compromising the prognostic and predictive value of axillary staging

\section{AIM OF THE WORK}

The aim of this work was to primarly evaluate the effect of neoadjuvant chemotherapy on axillary nodal status. The secondry objective was to evaluate percentage of patients who are eligible for SLNB post neoadjuvant chemotherapy.

\section{SUBJECTS AND METHODS}

\section{Patients:}

This retrospective study was conducted by collecting data during the period from October 2019 to July 2021.The study included sixty four patients who had biopsy proven locally advanced breast cancer with clinically or radiologically positive axillary LNs, had been received neoadjuvant chemotherapy at clinical oncology department and underwent surgery post neoadjuvant therapy at surgical oncology unit at Alexandria Main University Hospital.

\section{Exclusion criteria:}

Early breast cancer patients.

- Metastatic Stage IV breast cancer patients.

- Unfit patients for neoadjuvant therapy.

- Patients refusing neoadjuvant therapy.

Patients aged $<18$ years or $>75$ years.

-Patients with clinically and radiologically negative axilla.

\section{-Methods:}

Data were collected retrospectively to assess the oncological and surgical outcome after completing NAC

A total number of 450 patients files were reviewed and only sixty four patients were eligible patients to be included in our study.

\begin{tabular}{|c|c|c|c|c|}
\hline \multicolumn{4}{|c|}{ RESULTS } & \\
\hline \multicolumn{5}{|c|}{$\begin{array}{l}\text { Table : Results correlation between ypN0 and post } \\
\text { neoadjuvant axillary ultrasound } \mathrm{n}(64)\end{array}$} \\
\hline $\begin{array}{l}\text { Ultrasound axilla } \\
\text { postneoadjuvant }\end{array}$ & $\mathbf{N}$ & $\mathrm{ypN}_{0}$ & $\chi^{2}$ & ${ }^{\mathrm{MC}} \mathbf{p}$ \\
\hline Normal & 45 & $20(44.4 \%)$ & \multirow{3}{*}{$\begin{array}{c}10.005 \\
*\end{array}$} & \multirow{3}{*}{$\begin{array}{c}0.004 \\
*\end{array}$} \\
\hline Suspicious & 8 & $1(12.5 \%)$ & & \\
\hline Malignant & 11 & $0(0.0 \%)$ & & \\
\hline
\end{tabular}

$\chi^{2}$ : Chi square test, MC: Monte Carlo

$\mathrm{p}: \mathrm{p}$ value for comparing between the studied categories

*: Statistically significant at $\mathrm{p}<0.05$

\section{CONCLUSION}

According to our study we concluded that :

Post neoadjuvant ultrasound is essential for assessment of axillary response.

Around $44.4 \%$ of patients with negative post neoadjuvant axillary ultrasound can avoid unnecessary axillary LN clearance.

Accurate axillary staging is the corner stone for omission of axillary clearance for post neoadjuvant patients.

\section{RECOMMENDATIONS}

- Further prospective studies with large sample sizes to assess the false negative rate and feasibility of sentinel LN biopsy post neoadjuvant chemotherapy.

Application of sentinel LN biopsy for patients showing pos neoadjuvant clinical and radiological axillary response if we can reach a technique with an accepted false negative rate for SLNB in post neoadjuvant patients.

To assure the success of the procedure and increase the sensitivity of the SLNB:

- Dual technique

- Removal of three or more lymph nodes

- Clipping of the pathological LN before starting neoadjuvant and this clipped node is confirmed to be removed at the time of SLN biopsy.

$$
\begin{gathered}
\text { 20210Alexandria Faculty of Medicine } \\
\text { CC-BY-NC }
\end{gathered}
$$

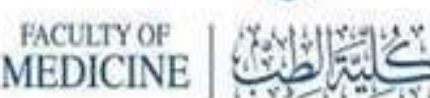

